\title{
¿Juega el locus de control de la salud un papel clave en la salud durante la adolescencia?
}

\author{
N. Zdanowicz MD \\ P. Janne Prof Psychology \\ CH. Reynaert Prof Psychiatry \\ Neurotec Department, Division of \\ Psychiatry, Karolinska Institutet, Huddinge \\ University Hospital, M-57, S-14186 \\ Stockholm
}

SWEDEN

\begin{abstract}
RESUMEN - Objetivos: Examinar las diferencias en el locus de control de la salud en una población de jóvenes "sanos" entre 13 y 25 años de edad y en una población con trastornos mentales.

Método: 814 jóvenes "normales" completaron el cuestionario sobre el locus de control de la salud y se compararon con una población de 358 jóvenes que padecían trastornos mentales.

Resultados: Los jóvenes del grupo sano obtuvieron una puntuación del locus de control de la salud "interno" (internal health locus of control, LCSI) más alta, y una ratio "interno" versus "externo" (I/E) más alta que los del grupo "enfermo". Por otra parte, las puntuaciones para la escala del locus de control de la salud de los "otros con poder" (powerful others health locus of control scale, LCSP) y para la escala del locus de control de la salud "por azar" (chance health locus of control scale, LCSA) fueron más altas en el grupo "enfermo". Mientras que la edad sólo influenció el locus de control de la salud en el grupo "sano", el género jugó un papel determinante en ambos grupos, en términos del LCSI.

Conclusiones: Los adolescentes que padecen trastornos mentales tienen un nivel de interiorización más bajo en el locus de control de la salud multidimensional (LCSM), que se expresa por un menor sentido de responsabilidad personal y un alto nivel de dependencia de los demás.
\end{abstract}

\section{Introducción}

En 1978, K.A. Wallston y colaboradores desarrollaron la escala del locus de control de la salud multidimensional (LCSM), que investigaba las creencias de una persona en relación al control que creía tener para determinar su estado de salud. Esta escala es multidimensional, de manera que identifica tres tipos distintos de creencias: dos "exter- 
nas" y una "interna". Los individuos con control interno creen que el bienestar que reciben depende de su comportamiento. Ésta es la escala del locus de control interno (LCSI). Los individuos "externos" creen que su salud es resultado bien de la suerte o del destino (subescala del locus de control de la salud "por azar", LCSA) o bien de la acción de terceras partes (subescala del locus de control de la salud de los "otros con poder", LCSP). Desde la creación de esta escala, numerosos trabajos, incluyendo una revisión llevada a cabo por A. Pauwels (1999) han mostrado, con fines principalmente preventivos primarios y secundarios de los individuos, que el LCSM es un buen predictor del estado de salud, tanto en medicina como en psiquiatría. Sin embargo, como todas las escalas, el LCSM tiene sus limitaciones. En particular, el LCSM parece ser menos relevante cuando se investiga el deseo de control y el valor atribuido por el individuo a la salud.

Que nosotros sepamos, sólo dos investigadores entre todos estos estudios han aplicado el LCSM en adolescentes. El estudio de W.R. Stanton (1995) investigó la utilidad y estabilidad del LCSM en la adolescencia. Además de confirmar la utilidad del LCSM, el estudio también resaltó las diferencias de género en el locus de control. Estos autores encontraron un cambio en las escalas LCSA y LCSP entre los 13 y los 15 años de edad, pero sólo en chicas. El segundo estudio, de S. Nada-Raja (1994), se llevó a cabo en un grupo de más de 800 jóvenes de 15 años de edad. Esta investigación no estudió, por tanto, la influencia de la edad en el LCSM, pero sacó a la luz las diferencias de género en las escalas del LCSI y LCSP.

La evolución del LCSM con la edad, sin embargo, nos pareció suficientemente importante para garantizar investigaciones futuras. Así, en dos estudios previos (Zdanowicz
2002, 2003 en prensa) y en un seguimiento del trabajo de Stanton, pudimos documentar en una muestra "sana" que, a pesar de que el LCSI no cambió con la edad, la ratio I/E (en otras palabras, internalización relativa como definió Renaert, 1995) aumentó ( $\mathrm{r}=0,0835$, $\mathrm{p}=0,027)$ y la puntuación LCSP disminuyó tanto en chicos como en chicas $(r=-0,1675$, $p<0,000)$. Con esta observación, una cuestión obvia que surge es si estos hallazgos se mantienen también para los adolescentes "enfermos" y si hay diferencias en el LCSM entre los grupos "enfermo" y "sano".

La respuesta a estas preguntas podría tener implicaciones para los distintos sectores involucrados en el cuidado de los adolescentes. De hecho, si las respuestas a estas dos preguntas son positivas, habríamos identificado aparentemente uno de los factores determinantes de la salud de los jóvenes. Así, no sólo tendríamos un instrumento a nuestra disposición para el que es posible imaginar varias aplicaciones preventivas (primarias, secundarias e incluso terciarias) sino que, posiblemente también, se trataría de un parámetro psicológico sobre el que se podrían basar actividades psicoterapéuticas. $\mathrm{Si}$, por ejemplo, examinamos el campo del trasplante de órganos en niños, en el que M.L. Stubher (1993) llega incluso a decir que la adherencia de un niño a un programa de trasplante depende primariamente de sus padres, podemos preguntar qué acciones y cuándo deberían tomarse para que el adolescente adquiera responsabilidad (Zdanowicz 1998).

Así, nuestras hipótesis se pueden formular de dos formas diferentes:

Una situación dinámica donde hay un relativo retraso en la internalización con la edad en una muestra "enferma", cuando se compara con una población "sana" de adolescentes, que se expresa en la muestra "enferma" mediante: 
1a) Un aumento más lento (o no aumento) en la ratio $\mathrm{I} / \mathrm{E}$.

1b) Una disminución más lenta (o no disminución) en la puntuación LCSP.

1c) Un aumento más rápido (o no aumento) en la puntuación LCSA.

Una situación donde el retraso da lugar a diferencias significativas entre la muestra "sana" y la muestra "enferma", que se expresa en la última mediante:

2a) Una ratio I/E más baja.

2b) Una puntuación LCSP más alta.

2c) una puntuación LCSA más alta.

Además, puesto que sabemos por estudios anteriores que la edad no influencia el LCSI en una muestra normal, podemos añadir dos hipótesis más:

1d) No hay relación entre la edad y la puntuación LCSI en la muestra "enferma".

2d) Sin embargo, la puntuación LCSI es más baja en la muestra "enferma".

Este estudio se llevó a cabo para responder, por lo menos, a estas cuestiones.

\section{Método}

Este estudio se llevó a cabo entre diciembre de 1998 y junio de 1999. Además del LCSM, los sujetos también describieron sus familias usando la tercera versión del cuestionario circunflejo de Olson (Olson 1985). Se incluyó esta valoración suplementaria para poder correlacionar el LCSM con los lazos familiares en un estudio futuro, siempre que nuestras hipótesis se confirmasen.

\section{Participantes}

Para clasificar los sujetos en el grupo "sano" o "enfermo", los candidatos tuvieron que reunir los siguientes criterios:

1. Tener entre 13 y 25 años de edad.

2. Estado civil: soltero o pareja (no matrimonio).

3. Estado socio-profesional: desempleado, sin profesión y/o estudiante.

Estos tres criterios son los propuestos por la Organización Mundial de la Salud (OMS 1998) para describir la "situación del adolescente”. Además, para asegurar la homogeneidad de nuestra población, los sujetos tenían que ser estudiantes, caucásicos y franco-parlantes.

\section{Procedimiento}

El grupo "sano"

El grupo "sano" se reclutó de dos formas distintas para asegurar diferentes grupos de edad. El primer reclutamiento se efectuó en cada uno de los seis años de educación secundaria general (en otras palabras, generalmente entre 12 y 18 años de edad) en tres colegios de secundaria diferentes en la provincia de Namur, Bélgica. Les pedimos a los jóvenes, en presencia de un psiquiatra evaluador, que proporcionaran información sociodemográfica (edad, nombre, género, nivel de educación, nacionalidad) y que completasen el LCSM y la escala de Olson para su familia y para su familia ideal. El primer reclutamiento lo llevó a cabo un grupo de estudiantes universitarios de cuarto año de Psicología en la Universidad Católica de Lovaina (UCL), que pidieron a sus compañeros que completaran un cuestionario anónimo (que incluía la fecha de nacimiento y el género), el LCSM y los cuestio- 
narios de Olson. Por defecto, nuestro grupo "sano" se considera "normal". Sin embargo, aunque este grupo no fue reclutado en una consulta u hospital, no podemos descartar que algunos de estos controles jóvenes estén en tratamiento médico o hayan sido hospitalizados por una u otra razón. Para minimizar esto, y debido a la frecuencia de trastornos depresivos a esta edad, los jóvenes completaron la escala autoadministrada para los sentimientos depresivos de Zung (1965). 10 sujetos pasaron el punto de corte de 0,699 (sospecha de estado depresivo) y, por tanto, fueron excluidos del protocolo.

\section{El grupo "enfermo"}

El grupo de adolescentes "enfermo" se constituyó utilizando datos codificados sistemáticamente desde 1990 procedentes de los pacientes hospitalizados en los departamentos de medicina psicosomática y psicopatología en las consultas de la UCL Mont-Godinne. La inclusión finalizó en diciembre de 1998. Al ingreso y bajo la supervisión de un psicólogo, los pacientes rellenaron sistemáticamente datos generales (incluyendo edad y fecha de nacimiento), un cuestionario LCSM y un cuestionario de Olson. Para todos los pacientes se trataba de la primera hospitalización, voluntaria, y los pacientes no procedían de ningún servicio de cuidados tipo "residencial". En un intento de controlar el efecto de la duración del período de inclusión, la fecha del ingreso se controló como co-variable.

Elegimos no tener en cuenta el diagnóstico para mantener la naturaleza trans-nosográfica de nuestras hipótesis. Sin embargo, para la primera hipótesis hemos controlado nuestros resultados para los dos diagnósticos más importantes ("trastornos depresivos" y "más de un diagnóstico").

\section{Análisis}

Los análisis estadísticos se llevaron a cabo usando el programa informático SPSS para Windows 95/98/NT, Modelos Avanzados 9.0. A la vista del importante número de sujetos y de la necesidad de revisar ciertas co-variables, utilizamos tests estadísticos paramétricos. Se utilizó el test $\mathrm{Chi}^{2}$ de Pearson para comparar las proporciones. Las correlaciones entre variables constantes se

Tabla I

Diagnósticos para el Eje I del DSM IV (1994)

\begin{tabular}{lrc} 
Eje I & $\mathrm{N}$ & $\%$ \\
\hline Número de diagnósticos para 358 adolescentes & 464 & 100 \\
Sin diagnóstico & 30 & 8,20 \\
Más de un diagnóstico & 121 & 33,8 \\
Trastornos depresivos & 179 & 37 \\
Trastornos de ansiedad & 71 & 14,7 \\
Trastornos por consumo de alucinógenos & 64 & 13,2 \\
Trastornos por déficit de atención y conducta desordenada & 49 & 10,1 \\
Esquizofrenia y otros trastornos psicóticos & 27 & 5,6 \\
Trastornos de conducta alimentaria & 24 & 4,9 \\
Trastornos por consumo de alcohol & 23 & 4,8 \\
Trastornos somatomorfos & 18 & 3,7 \\
Trastornos de adaptación & 9 & 1,8 \\
Trastornos del aprendizaje & 8 & 1,6 \\
Trastornos por consumo de varias sustancias adictivas & 7 & 1,4 \\
Trastornos facticios & 1 & 0,2 \\
Retraso mental & 1 & 0,2
\end{tabular}


evaluaron empleando el coeficiente $r$ de Pearson y, si era necesario, revisando para la co-variable (correlación parcial). Las variables cuantitativas se compararon mediante un ANOVA ponderado para las co-variables. Los límites de significación son: tendencia, a nivel de $\mathrm{p}<0,1$, significación a nivel de $\mathrm{p}<$ 0,05 y alta significación a nivel de $\mathrm{p}<0,01$. Todos los análisis estadísticos se realizaron considerando las 2 colas de la distribución.

\section{Presentación de resultados}

Los resultados se presentan en el siguiente orden: análisis de parámetros demográficos (edad y género), discusión sobre el impacto de esos parámetros, análisis sucesivos de las hipótesis.

Los otros parámetros demográficos clásicos, tales como el origen étnico, la ocupación y el nivel de educación, no son relevantes en este estudio. El origen étnico y la ocupación son constantes, debido al criterio de inclusión.

\section{Resultados}

\section{Características demográficas}

\section{Distribución por edad}

El grupo "sano" se compone de 814 sujetos de entre 13 y 25 años de edad, con una edad media de 18 años y una desviación estándar de 3 años. El grupo "enfermo" incluyó 358 sujetos, con una edad media de 20,5 años (rango: 14 a 25 años) y una desviación estándar de 3 años. Estas diferencias son estadísticamente significativas $(\mathrm{t}=-13,179$, $\mathrm{p}=0,000)$.

\section{Distribución por género}

La ratio por género fue de 329 hombres y 436 mujeres $(0,75)$, en el grupo "sano", y
129 hombres y 229 mujeres en el grupo "enfermo" (ratio $=0,56$ ). Estas diferencias son estadísticamente significativas $\left(\mathrm{Chi}^{2}=\right.$ $69,112, \mathrm{p}=0,000)$.

\section{Impacto de los parámetros demográficos}

Ya que las diferencias en la variable "edad" son significativas entre los dos grupos, este parámetro debería tenerse en cuenta en el análisis estadístico cuando se comparan ambos grupos. Sin embargo, ya que las hipótesis 1a) a 1d) son hipótesis correlativas formuladas como una función de edad, revisamos este factor de todas formas. Este no es el caso para la variable "género" que, debido a que las diferencias entre los dos grupos son también significativas, debería revisarse sólo si el resultado es positivo.

\section{Verificación de las hipótesis}

\section{Hipótesis 1:}

Hay un retardo en la internalización con la edad en la muestra "enferma" de adolescentes, comparado con la muestra "sana", que se caracteriza en el primero mediante:

1a) Un aumento más lento (o no aumento) en la ratio $\mathrm{I} / \mathrm{E}$.

1b) Una disminución más lenta (o no disminución) en la puntuación LCSP.

1c) Un aumento más rápido (o no aumento) en la puntuación LCSA.

1d) No hay relación entre la edad y la puntuación LCSI en la muestra "enferma" tampoco.

Como se ilustra en la Tabla II, se confirman claramente estas hipótesis. De hecho, cuando se examinan los puntos de corte, no se puede encontrar correlación. De este modo, en los sujetos "enfermos" estos parámetros no cambian (o no cambian más) con 
la edad, como si la evolución "normal" se hubiera bloqueado. Además podemos afirmar que, en los casos de los "deprimidos" y de los "de más de un diagnóstico", hay una estabilidad del LCSI e incluso de la ratio $\mathrm{I} / \mathrm{E}$, con la edad.

\section{Hipótesis 2:}

El retraso en la internalización relativa da lugar a diferencias significativas entre la muestra "sana" y la muestra "enferma", que se expresan en ésta última como sigue: 2a) Una ratio I/E más baja.

2b) Una puntuación LCSP más alta.

2c) Una puntuación LCSA más alta.

2d) Una puntuación LCSI más baja.

Todas estas hipótesis se confirmaron mediante un ANOVA (con LCSM y género como variables y edad como co-variable), como se demuestra en la Tabla III. Excepto para el LCSI, donde se evidencia un efecto de la variable "género", las otras diferencias no parecen significativas.

Tabla II

Correlación de los parámetros del locus de control de salud multidimensional (LCSM) con la edad

\begin{tabular}{lcccc} 
& I/E & LCSP & LCSA & LCSI \\
\hline GRUPO "ENFERMO", & $\mathrm{N}=354$ & $\mathrm{~N}=354$ & $\mathrm{~N}=354$ & $\mathrm{~N}=354$ \\
Coeficiente r de Pearson & $-0,006$ & 0,106 & $-0,16$ & 0,083 \\
$\mathrm{p}$ & 0,964 & 0,428 & 0,237 & 0,521 \\
TRASTORNOS DEPRESIVOS & $\mathrm{N}=171$ & $\mathrm{~N}=171$ & $\mathrm{~N}=171$ & $\mathrm{~N}=171$ \\
Coeficiente r de Pearson & 0,0012 & 0,0215 & $-0,0014$ & 0,0089 \\
p & 0,988 & 0,781 & 0986 & 0,909 \\
MÁS DE 1 DIAGNÓSTICO & $\mathrm{N}=121$ & $\mathrm{~N}=121$ & $\mathrm{~N}=121$ & $\mathrm{~N}=121$ \\
Coeficiente r de Pearson & $-0,051$ & 0,1365 & 0,0256 & $-0,004$ \\
$\mathrm{p}$ & 0,563 & 0,119 & 0,775 & 0,996 \\
\hline
\end{tabular}

Tabla III

Comparación del LCSM en los grupos "sano" y "enfermo". ANOVA controlado para la edad (co-variable)

\begin{tabular}{lrccrrr} 
LCSM & $\mathrm{F}$ & $\begin{array}{c}\mathrm{N} \\
\text { (sano-enfermo) }\end{array}$ & $\begin{array}{c}\text { Media } \\
\text { (sano-enfermo) }\end{array}$ & $\begin{array}{c}\text { d.e. } \\
\text { (sano-enfermo) }\end{array}$ & $\begin{array}{c}\mathrm{p} \\
\text { (intercept) }\end{array}$ \\
\hline I/E & 19,205 & $740-354$ & $1,40-1,12$ & $0,5-0,4$ & $<0,000$ & $<0,000$ \\
Chicas : & 1,049 & $429-226$ & $1,37-1,08$ & $0,51-0,39$ & 0,111 & $<0,000$ \\
Chicos: & & $311-128$ & $1,44-1,19$ & $0,57-0,42$ & & \\
LCSP & 37,516 & $740-355$ & $19,2-22,3$ & $5,8-6,3$ & $<0,000$ & $<0,000$ \\
Chicas : & 2,862 & $429-226$ & $18,9-21,9$ & $5,3-5,6$ & 0,058 & $<0,000$ \\
Chicos : & & $311-128$ & $19,5-22,9$ & $5,0-6,1$ & & $<0,000$ \\
LCSA & 15,501 & $740-354$ & $17,9-19,9$ & $5,5-5,8$ & $<0,000$ & $<0,000$ \\
Chicas : & 2,941 & $429-226$ & $18,2-20,7$ & $5,3-5,6$ & 0,053 & $<0,000$ \\
Chicos : & & $311-128$ & $17,5-19,8$ & $5,7-6,2$ & & $<0,000$ \\
LCSI & 9,589 & $740-354$ & $24,1-22,6$ & $4,7-6,1$ & $<0,000$ & $<, 022$ \\
Chicas : & 3,829 & $429-226$ & $23,7-21,7$ & $4,7-5,6$ & 0,0 & \\
Chicos : & & $311-128$ & $24,6-24,2$ & $4,8-6,5$ & & \\
\hline
\end{tabular}




\section{Discusión}

Nuestro estudio da lugar a tres conceptos que surgen de las observaciones hechas por Stanton (1995) y Zdanowicz (2002, 2003 en prensa) en los adolescentes "sanos":

1) El sentido de responsabilidad hacia la salud (LCSI) parece, en la adolescencia, haber sido determinado ya antes de alcanzar la misma, como si no cambiase con la edad ni en sujetos "sanos" ni en "enfermos". Sin embargo, es más bajo en los adolescentes "enfermos" que en los "sanos".

2) La evolución descrita en los sujetos "sanos", que incluye un incremento en la internalización relativa, la dimensión de "azar" y la disminución en la dimensión de los "otros con poder" con la edad, no ocurre en los adolescentes "enfermos". Además, los sujetos "enfermos" tienen niveles más altos de "azar" y de los "otros con poder", y un nivel más bajo de internalización relativa. Esto significa que la gente joven "enferma" espera más de los otros y del azar (o del destino), y creen menos en su propia capacidad para escapar de la situación. Se pueden exponer tres teorías para explicar esta carencia en la evolución:

- O bien hay una fragilidad durante el desarrollo respecto a la posibilidad de "caer enfermo". De hecho, no podemos imaginar que este fenómeno sea otra cosa que algo propio del desarrollo, y S. Nada-Raja (1994) ha demostrado ya la influencia de las creencias maternas sobre las chicas, así como la influencia de factores fácticos o constitucionales en los chicos.

- O el estado de enfermedad lleva a que los jóvenes tengan falta de confianza en sí mismos y dependan de las influencias externas, particularmente al principio de la hospitalización.
- O hay combinaciones de estas dos explicaciones como "cuanta más falta de confianza en mí mismo y más dependencia tengo de los demás, más susceptible soy de ponerme enfermo, tengo menos confianza en mí mismo y dependo más de los demás".

Puesto que no se ha realizado ningún estudio prospectivo usando el LCSM (aparte del realizado por P.D. Thomas en 1983, pero en el que no se estudia su relación con la enfermedad, sino con la probabilidad de integración social de los ancianos durante un periodo de cinco años como una función de su LCSM), es ciertamente difícil confirmar que la primera explicación ha sido validada a fondo. Por tanto, la tercera explicación nos parece la más probable.

3) Finalmente, está la actitud de alguna manera paradójica vista en los chicos "enfermos", que continúan creyendo que están en una posición de poder respecto a su salud.

\section{Limitaciones y conclusión}

La principal restricción relativa a la validez de nuestros resultados es ciertamente la duración del reclutamiento de la muestra "enferma". De hecho, analizar los datos controlándolos para la fecha de hospitalización no nos permite excluir totalmente parámetros socioculturales, farmacológicos $\mathrm{u}$ otras modificaciones que podrían haber influenciado nuestros resultados.

Sin embargo, a la luz de estas observaciones, creemos que este estudio podría tener diferentes implicaciones prácticas (que por supuesto tienen que ser sometidas a prueba en un diseño prospectivo). Por ejemplo, quizá nos permitiría predecir el riesgo de adicción al tabaco o al alcohol en un adoles- 
cente que todavía está en periodo de empezar a probar estas sustancias.

Este estudio, aparentemente, también devuelve a los trastornos mentales en los adolescentes una perspectiva evolutiva que recordaría la idea de los años 80 de que una crisis de adolescencia es equivalente a una crisis de salud. Aunque, ciertamente, ya no se defiende la idea de que la sintomatología psíquica en la adolescencia es un signo de salud mental, nuestro estudio, sin embargo, valida las observaciones hechas por P. Male (1982) y M. Laufer (1975). Estos autores defendieron la necesidad de una nosografía del desarrollo. Esta forma de construir la nosografía dirige la patología juvenil hacia un concepto clínico de patologías evolutivas más que hacia un enlentecimiento evolutivo, que es la tendencia actual. Sin embargo, se requerirán estudios futuros para identificar los determinantes (quizá familiares) que podrían enlentecer este desarrollo.

\section{Bibliografía}

DSM IV. Diagnostic and Statistical manual of mental disorders. Fourth edition. Washington, American Psychiatric Association Press, 1994.

LAUFER, M. Troubles psychiques chez les adolescents. Paris, Le Centurion, 1975.

MÂLE, P. La crise juvénile. Paris, Payot, 1982.

NADA-RAJA, S., McGEE, R., WILLIAMS, S. Health beliefs among New Zealand adolescents. Journal of Paediatrics and Child Health; 30: 523-529, 1994.

OLSON, D.H., KILLORIN, E. Clinical rating scale for circumplex model (revised version). St Paul MN: Family Social Science: University of Minnesota Press, 1985.

ORGANISATION MONDIALE DE LA SANTÉ (WHO). La santé des jeunes. Genève, OMS, 1998.
PAUWELS, A., JANNE, P., REYNAERT, CH. De différents modèles de croyences envers la santé au vécu subjéctif de contrôle vis à vis de la santé: une tentative d'approche intégrative. Journal de Thérapie Comportementale et Cognitive, 9: 99-107, 1999.

REYNSERT, CH., JANNE, P., DELIRE, V. To control or to be controlled? From health locus of control to morphine during pateint-controlled analgesia. Psychotherapy and Psychosomatic, 319: 1-8, 1995.

THOMAS, P.D., HOOPER, E.M. Healthy elderly: social bonds and locus of control. Research in Nursing Health, 6: 11-16, 1983.

STANTON, W.R., NADA-RAJA, S., LANGLEY, J. Stability in the structure of health locus of control among adolescents. Britisch Journal of Clinical Psychology, 34: 279-287, 1995.

STUBHER, M.L. Psychiatric aspects of organ transplantation in children and adolescents. Psychosomatic, 34: 379-389, 1993.

WALLSTON, K.A., WALLSTON, B.S., DE VELLIS, R. Development of the multidimensional health locus of control (MHLC) scale. Health Educating Monography, 6: 160-170, 1978.

ZDANOWICZ, N, REYNSERT, CH., JANNE, P. Transplants and psychiatry. Psychosomatic, 39: 390-391, 1998.

ZDANOWICZ, N., JANNE, P., REYNAERT, CH. Comparing family expectancies of "healthy" and "pathological" students. Annale Médico-Psychologique, 160: 130137, 2002.

ZDANOWICZ, N., JANNE, P., REYNAERT, CH. L'adolescent est-il responsable de sa santé. European Journal of Psychiatry in press.

ZUNG, W.K. A self rating depression scale. Archives General Psychiatry, 12: 63-70, 1965.

Dirección para correspondencia:

N. Zdanowicz

Université Catholique de Louvain

Clinique de Mont-Godinne

Service de psychosomatique

5530 Yvoir

BELGIQUE - BELGIUM

E-mail: nicolas.zdanowicz@pscl.ucl.ac.be 